\title{
Determination of sdLDL particles in patients with Familial Hypercholesterolaemia and Familial Combined Hyperlipidaemia
}

$\mathrm{BioFIG}$

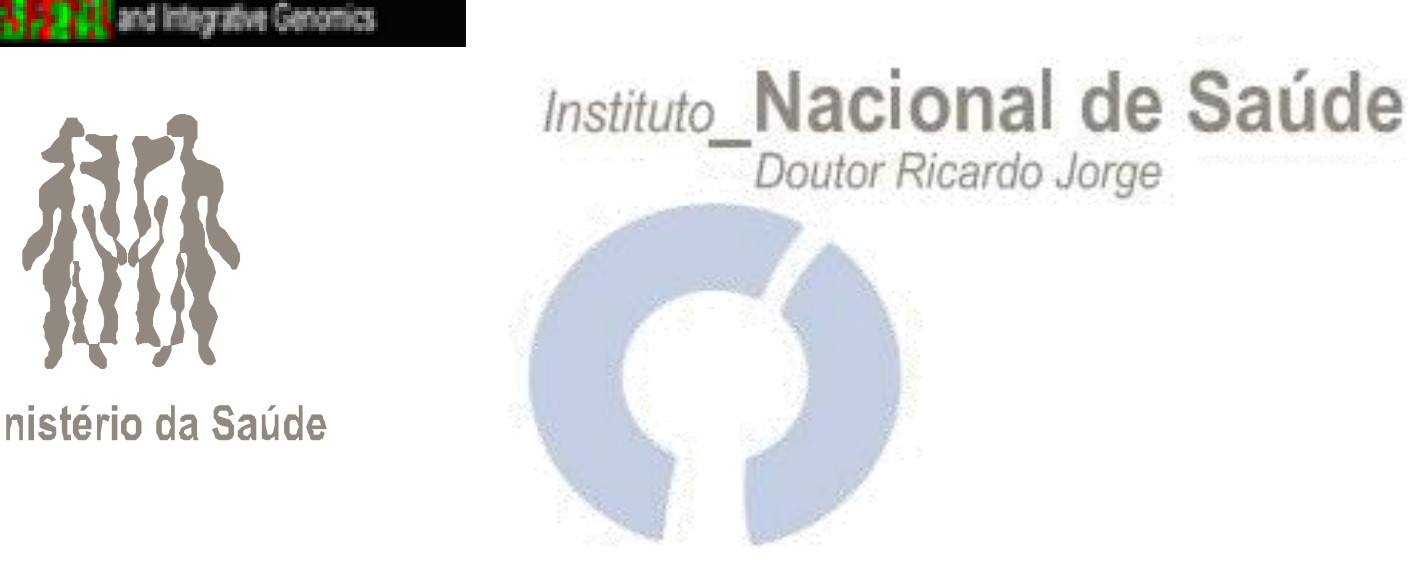

A. Gomes $^{1,2}$, T. Santos ${ }^{1,2}$, M. Bourbon ${ }^{1,2}$

1 Grupo de Investigação Cardiovascular, DPSDC, Instituto Nacional Saúde Dr Ricardo Jorge, Lisboa, Portugal 2 Center for Biodiversity, Functional \& Integrative Genomics (BIOFIG)

mafalda.bourbon@insa.min-saude.pt

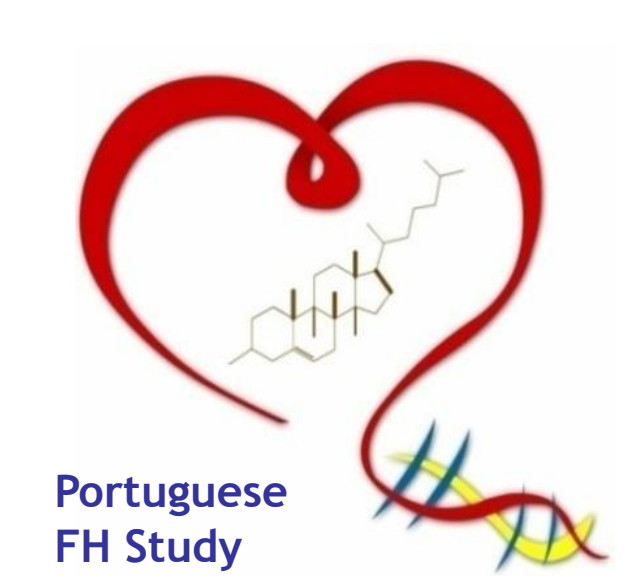

\section{Background}

Cardiovascular disease (CVD) is a multifactorial disorder depending on both genetic and environmental factors. Low density lipoprotein (LDL) comprises a heterogeneous population of particles in terms of size, biochemical properties and atherogenic potencial. Several studies have demonstrated that small dense LDL (sdLDL) particles are an emerging cardiovascular (CV) risk factor due to its atherogeneicity. The objective of this study was the measurement of sdLDL in patients with genetic diagnosis of Familial Hypercholesterolaemia $(\mathrm{FH})$ and clinical diagnosis of Familial Combined Hyperlipidaemia (FCHL) to establish a relation between sdLDL, CV risk and the efficacy of therapeutics.

\section{Methods}

Lipid profile was determined by the measurement of LDL particle size and its cholesterol concentration using a polyacrylamide gel electrophoresis method (Lipoprint system, Quantimetrix) that separates the LDL subfractions present in fasting serum samples. The lipidogram obtained classifies the patients as being profile $\mathrm{A}$ (low $\mathrm{CV}$ risk) or B (high $\mathrm{CV}$ risk) depending mainly on the sdLDL concentration. Total, LDL and high density lipoprotein (HDL) cholesterol, apolipoprotein Al (ApoAI), ApoB, lipoprotein(a) and triglycerides were also measured in an automated analizer (Cobas 400, Roche).

\section{Results}

- The lipid profile was obtained from $43 \mathrm{FH}$ adults and $\mathbf{4 6} \mathrm{FCHL}$ adults, index and relatives.

- Significant results were found for patients without medication and with high sdLDL (>6mg/dl) where FH patients presented higher levels of total cholesterol, LDL and ApoB and FCHL patients presented higher levels of total cholesterol, LDL , ApoB and triglycerides (TG), compared to patients with sdLDL under recommended values (Fig. 1).

- Under medication FH patients have significant higher ApoB levels and lower HDL, and FCHL patients have significant higher total cholesterol, LDL and ApoB levels (Fig. 1).

- In Figure 2 there are presented some examples of lipidograms of the patients of this study obtained from the Lipoprint System.

- In the FCHL patients group that were on medication $71,4 \%$ still presented a high CV risk profile (profile B) (Fig. 3).
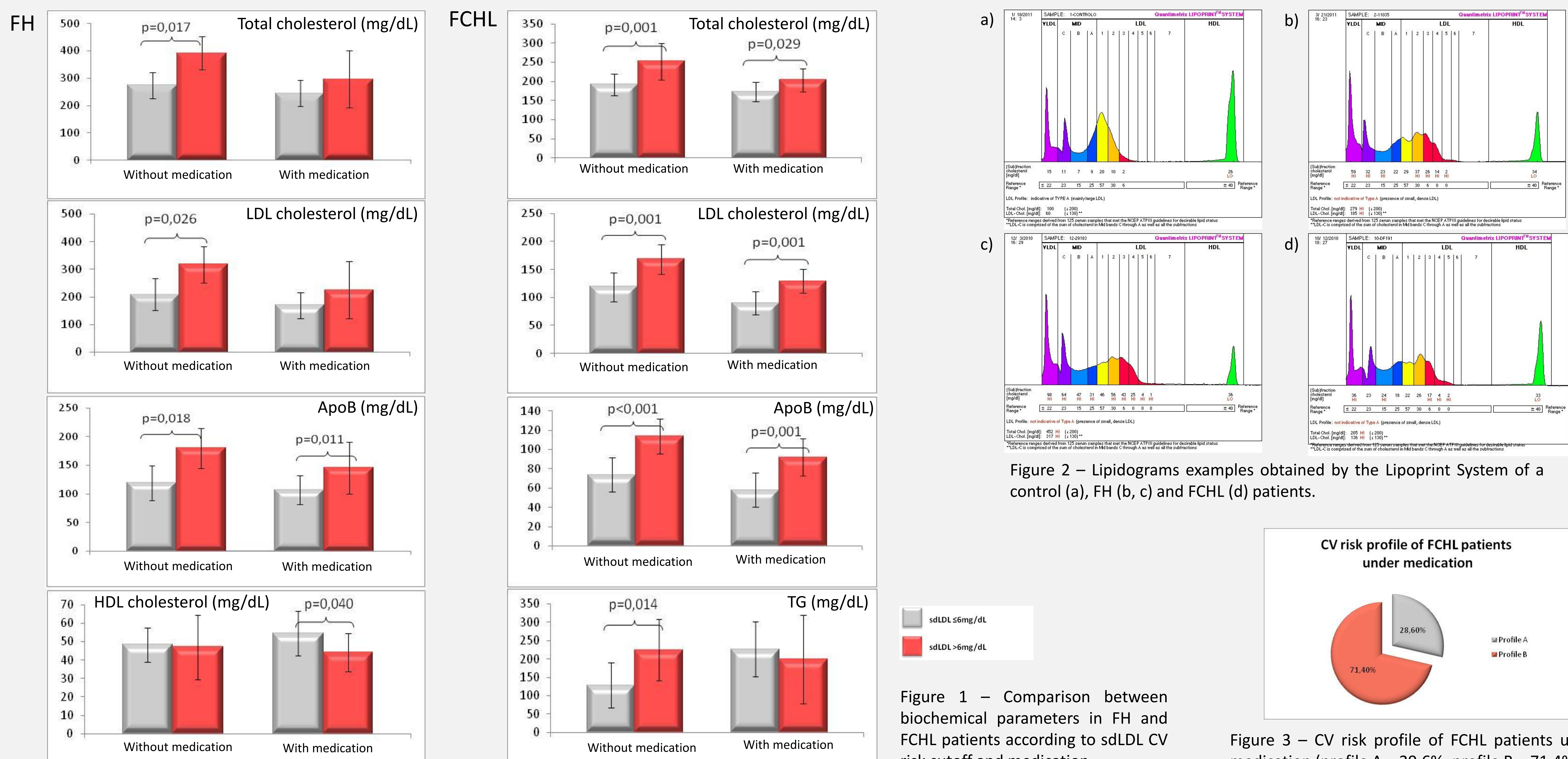

Figure 2 - Lipidograms examples obtained by the Lipoprint System of a control (a), FH (b, c) and FCHL (d) patients.

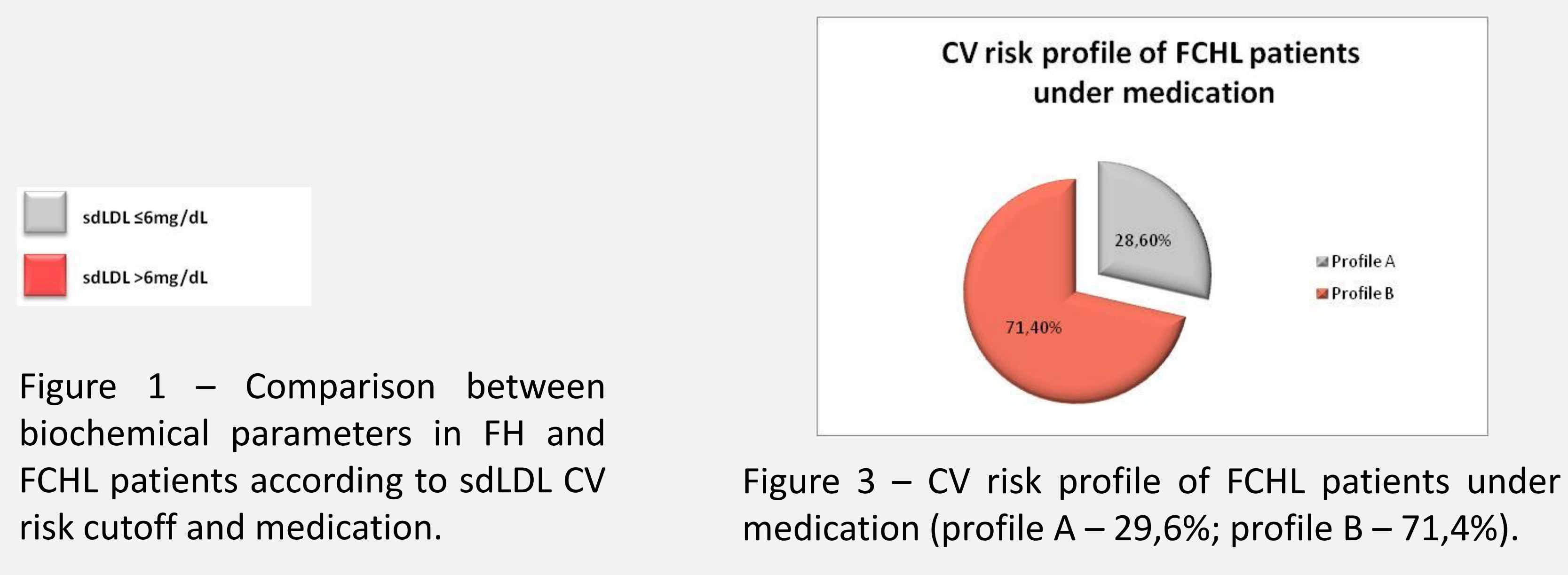

\section{Discussion and Conclusions}

Total, LDL and HDL cholesterol and also ApoB and TG levels seem to be differentially distributed between FH ad FCHL patients when taking into account the presence of sdLDL and the use of medication.

$71,4 \%$ of the FCHL patients under medication still presented a high CV risk profile, showing that statins seem not to decrease sdLDL levels and neither CV risk. Also FCHL patients are not well medicated or do not respond to usual medication to decrease cholesterol.

These preliminary results indicate that the measurement of sdLDL could be a good biomarker for treatment control but further studies are needed to evaluate the effect of medication in sdLDL levels in FH and FCHL patients. 